\title{
The Spatial Distribution of Attention Within and Across Objects
}

\author{
Andrew Hollingworth \\ The University of Iowa
}

\author{
Ashleigh M. Maxcey-Richard \\ Manchester College
}

\author{
Shaun P. Vecera \\ The University of Iowa
}

\begin{abstract}
Attention operates to select both spatial locations and perceptual objects. However, the specific mechanism by which attention is oriented to objects is not well understood. We examined the means by which object structure constrains the distribution of spatial attention (i.e., a "grouped array"). Using a modified version of the Egly et al. object cuing task, we systematically manipulated within-object distance and object boundaries. Four major findings are reported: 1) spatial attention forms a gradient across the attended object; 2) object boundaries limit the distribution of this gradient, with the spread of attention constrained by a boundary; 3 ) boundaries within an object operate similarly to across-object boundaries: we observed object-based effects across a discontinuity within a single object, without the demand to divide or switch attention between discrete object representations; and 4) the gradient of spatial attention across an object directly modulates perceptual sensitivity, implicating a relatively early locus for the grouped array representation.
\end{abstract}

Keywords: attention, object-based attention, spatial attention, grouped array

Attending within the visual world typically involves attending to objects. During the viewing of a natural scene, eye movements are directed, almost exclusively, to discrete objects and are rarely directed to empty regions of space (Henderson \& Hollingworth, 1999). During complex, everyday tasks, attention is directed sequentially to objects of immediate relevance for task execution (Land \& Hayhoe, 2001). Initial research on selection within the visual system focused on the selection of particular environmental locations, independently of the structure imposed by objects and surfaces (e.g., Posner, Snyder, \& Davidson, 1980). Subsequently, researchers have investigated whether, and how, object perceptual structure shapes and guides the allocation of visual attention (for reviews, see Luck \& Vecera, 2002; Mozer \& Vecera, 2005b; Scholl, 2001). Much of this work has been devoted to demonstrating that object structure does indeed modulate visual selection in various contexts. However, several lines of research have been devoted to explicating the specific mechanisms by which object selection occurs.

Research into the mechanisms of object-based attention has converged on the conclusion that object representations mediate attentional selection at multiple levels of the visual system and that object-based selection at these different levels is subserved by distinct mechanisms. First, researchers have proposed that at rel-

This article was published Online First July 4, 2011.

Andrew Hollingworth and Shaun P. Vecera, Department of Psychology, The University of Iowa; Ashleigh M. Maxcey-Richard, Department of Psychology, Manchester College.

Correspondence concerning this article should be addressed to Andrew Hollingworth, Department of Psychology, The University of Iowa, 11 Seashore Hall E, Iowa City, IA 52242-1407. E-mail: andrew-hollingworth@ uiowa.edu atively early stages of visual perception, the image structure of visible objects (such as contours and boundaries) shapes the distribution of spatial attention, a grouped-array hypothesis (Kramer \& Jacobson, 1991; Kramer, Weber, \& Watson, 1997; Vecera, 1994; Vecera \& Farah, 1994). Object boundaries limit the spread of spatial attention (Avrahami, 1999; Marino \& Scholl, 2005), with attention coming to "fill" the region bounded by the object. At the heart of this view is the claim that higher-level object structure feeds back into earlier visual areas, increasing perceptual sensitivity to stimuli falling within the spatial region defined by the object (see also Mozer, 2002). Evidence for this type of mechanism comes from studies showing that spatial manipulations alter the effect of objects on perceptual processing (Davis, Driver, Pavani, \& Shepherd, 2000; Kramer, Weber, \& Watson, 1997; Kravitz \& Behrmann, 2008; Vecera, 1994) and that object structure modulates spatial patterns of activation in early visual areas (N. G. Müller \& Kleinschmidt, 2003), influencing relatively early components of visual perception (He, Fan, Zhou, \& Chen, 2004; Martinez, Ramanathan, Foxe, Javitt, \& Hillyard, 2007; Martinez, Teder-Salejarvi, \& Hillyard, 2007; Martinez et al., 2006; ValdesSosa, Bobes, Rodriguez, \& Pinilla, 1998). Second, object structure may influence shifts of attention, with a shift in the spatial locus of attention either more efficient or more likely within an object than between objects (Egly, Driver, \& Rafal, 1994; Lamy \& Egeth, 2002). Third, strategic prioritization of within-object locations may occur when the position of a target is uncertain (Drummond \& Shomstein, 2010; Shomstein \& Yantis, 2002, 2004). Finally, higher-level object representations can be selected to some extent independently of object position (Duncan, 1984; Vecera \& Farah, 1994). Such spatially invariant selection most plausibly occurs over visual representations that are abstracted away from the precise metric structure of early vision, are maintained in brain regions that do not preserve precise spatial information (such as 
inferior temporal cortex), and depend on higher-level visual representational systems such as visual working memory. Indeed, recent evidence suggests that the object advantage in the original Duncan (1984) task, in which participants are more efficient at reporting the features of a briefly presented stimulus when the features belong to a single object, has its locus in object-based visual working memory representations (Awh, Dhaliwal, Christensen, \& Matsukura, 2001; Matsukura \& Vecera, 2009; Vecera \& Farah, 1994).

Given that object-based attention is likely to be composed of multiple distinct mechanisms of selection, it is necessary to isolate particular mechanisms for direct study. In the present set of experiments, we focused on the means by which object structure shapes the distribution of spatial attention. As discussed above, one theoretical account of this mechanism is the grouped array theory originally developed by Vecera and Farah (1994). In this view, attending to an object involves attending to a set of locations that have been grouped together by perceptual organization processes. Grouping processes organize visual features that are represented in a spatio-topic or array-format representation. These grouping processes then constrain spatial attention, with the spread of attention limited by the contours and boundaries of the attended object. Spatial attention is the selection mechanism in the grouped array account, and selection occurs by enhancing the perceptual processing of the locations comprising the attended object. In short, targets appearing on an attended object are proposed to be more perceptible than targets appearing elsewhere. In the spatial attention literature, perceptual enhancement effects are typically discussed as resulting from limited capacity attentional processes (Posner, 1980); when these resources are directed to a particular location, targets at that location are identified more efficiently than targets appearing at other locations.

The grouped array hypothesis provides a straightforward explanation of certain object-based attentional effects. In the objectbased cuing task developed by Egly, Driver, and Rafal (1994), perceptual enhancement of the set of locations corresponding to the attended object allows targets on an attended object to be detected more efficiently than those appearing on an unattended object. Perceptual enhancement can also explain results from flanker tasks (Chen \& Cave, 2006; Richard, Lee, \& Vecera, 2008; but see Shomstein \& Yantis, 2002). In Richard, Lee, \& Vecera (2008), the effect of flanker compatibility on response to a central target was greater when the flankers were presented within the same object as the target (compared with the effect of equidistant flankers presented in a different object). Under the grouped array account, attention spreads within an object, enhancing the perceptual processing of stimuli falling within the object, allowing within-object flankers to influence target processing more than flankers that do not perceptually group with the target.

\section{The Present Study}

In the present study, we sought to broaden the grouped array theory by investigating three issues central to understanding how object structure modulates the distribution of spatial attention.

The spatial distribution of attention within an object. Spatial attention is allocated in a graded manner across the visual field (LaBerge \& Brown, 1989). In cuing studies, perceptual processing is facilitated at the cued location, with perceptual efficiency grad- ually decreasing with increasing distance from the cue (Downing \& Pinker, 1985; Henderson, 1991; Henderson \& Macquistan, 1993; Mangun \& Hillyard, 1988). If object structure serves to constrain a spatial selective mechanism that is inherently graded, attention directed to objects should exhibit a spatial gradient across the object. Evidence of a nonuniform distribution of attention within an attended object has already been obtained in the Egly et al. (1994) paradigm: perceptual processing at the cued location within an object is more efficient than processing at other locations within the same object. However, previous studies have probed only two locations within the attended object, and thus could not establish whether such spatial effects reflect an underlying mechanism that is smoothly graded. In the present experiments, we probed multiple locations within an attended object to determine whether attention is indeed smoothly graded across the object and to map the shape of the gradient.

The role of object boundaries in constraining the spread of spatial attention. The grouped array theory holds that object boundaries are critical to constraining the spatial distribution of attention. In this view, any form of object boundary should block or retard the spread of attention, even if the discontinuity occurs within a single object (rather than between objects). Using ringshaped objects, we examined whether a gap in the object would retard the spread of attention, generating an "object-based" effect within a single object. To probe the extent to which local object discontinuities are responsible for object-based effects of attention in general, the effect of a gap in a single object was compared with the effect of an identical gap between two different objects. In addition, we examined the effect of an object gap on the shape of the spatial gradient of attention. Finally, we asked whether an object boundary completely blocks the spread of attention across a gap or just retards the spread of attention, with some "leakage" of attention across the boundary (Kravitz \& Behrmann, 2008).

Effects of the spatial distribution of attention within and across objects on perceptual sensitivity. Finally, we examined whether object-based selection affects the efficiency of perceptual processing (i.e., perceptual enhancement of locations enclosed by the object). The grouped array account holds that object structure shapes the spread of spatial attention, and spatial attention directly influences the efficiency of perceptual processing by enhancing sensory signals at relatively early levels of the visual system (Carrasco, Penpeci-Talgar, \& Eckstein, 2000; Henderson, 1996; Hillyard, Vogel, \& Luck, 1998; Luck, Hillyard, Mouloua, \& Hawkins, 1996; Pestilli \& Carrasco, 2005). Thus, effects of objects on attention should be observed using methods optimized to measure perceptual sensitivity.

\section{General Method}

The general method was built upon the object cuing paradigm developed by Egly et al. (1994), which has been shown to be sensitive to spatial manipulations (Vecera, 1994) and thus appropriate for examining the role of object structure in shaping the spatial distribution of attention. Our modified paradigm is illustrated in Figure 1. Each trial of the present method began with the presentation of two, semicircular "tube" objects rendered from three-dimensional (3D) models. One end of an object was cued by the appearance of a "bulge" that grew out of and receded into the object over $50 \mathrm{~ms}$ of animation. After a delay of $70 \mathrm{~ms}$, a discrim- 


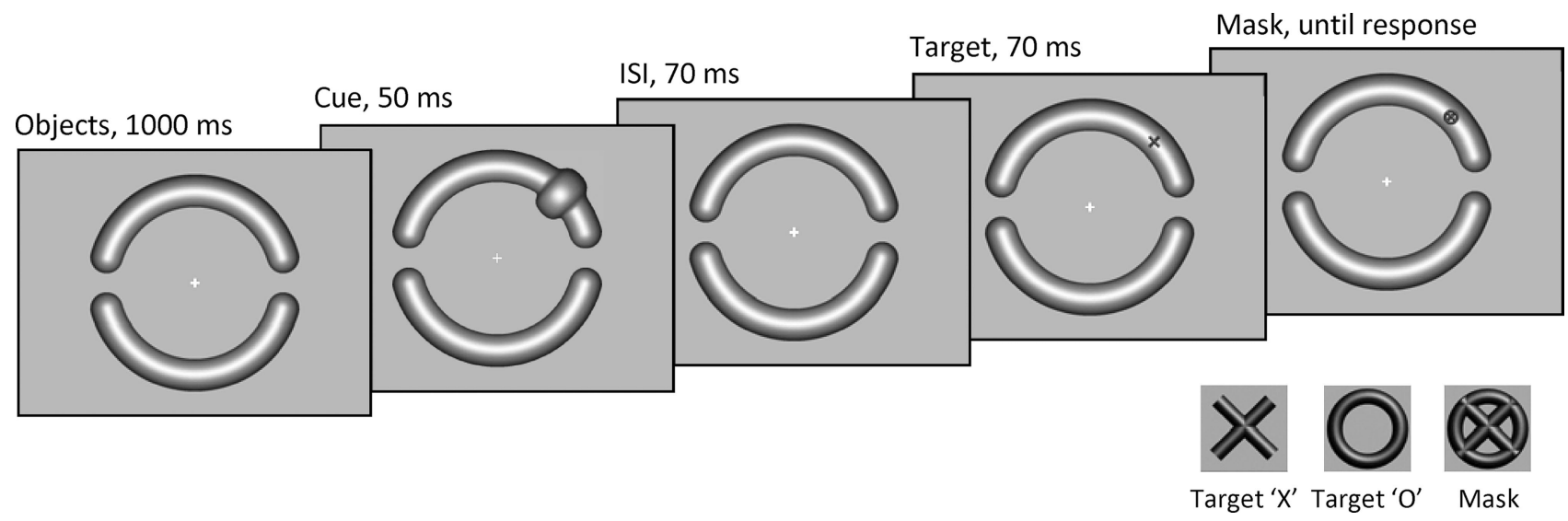

Figure 1. Sequence of events in a trial and target stimuli for the masked-discrimination paradigm. Inset stimuli show the two possible targets and the mask.

ination target was presented (an ' $\mathrm{X}$ ' or an 'O') for $70 \mathrm{~ms}$ before being masked by a composite X-O mask. Participants reported target identity, and discrimination accuracy was the primary dependent measure. The target appeared at the cued location or at an uncued location. The primary manipulations were the withinobject distance between cue and target (to probe gradient effects within the cued object), the object in which the cue and target appeared (same object or different object), and the presence of one versus two gaps (to assess the effect of local discontinuity in a single object on the gradient of attention and compare this with the effect of a between-object discontinuity).

The use of 3D-rendered objects provided strong shape and shading cues to discriminate the objects from the background and from each other (for a similar use of 3D cues, see Atchley \& Kramer, 2001). This was intended to maximize the potential effects of object structure on the distribution of spatial attention. Cues (bulge animation) and targets ( $\mathrm{X}$ and $\mathrm{O}$ ) appeared to be properties of the objects themselves, a feature designed to ensure that attention to and discrimination of object properties operated over features intrinsic to the objects. ${ }^{1}$ Semicircular objects (instead of the standard rectangles) were used so that all target locations on the objects were equally distant from central fixation, ensuring that manipulations of cue-target distance were not confounded with differences in eccentricity (e.g., Kwak, Dagenbach, \& Egeth, 1991).

In addition to these modifications of the original Egly et al. (1994) paradigm, our basic paradigm was designed to be sensitive to differences in perceptual sensitivity within an object and across an object boundary. Although the original onset detection paradigm of Egly et al. (1994) has often been interpreted as probing perceptual sensitivity, onset detection RTs can be strongly influenced by changes in response criteria (see Luck \& Vecera, 2002 for an extended discussion), and thus the onset detection paradigm is not ideal for the present purposes. For example, shorter RTs to within-object targets in the standard Egly et al. task could be generated if participants adopted a lower criterion for triggering a response to targets appearing within an object, without any effect of object on perceptual sensitivity per se. The masked discrimination procedure used here is to be preferred over onset detection, because it assesses perceptual sensitivity in a manner that is not influenced by criterion differences. For this reason, masked discrimination is the dominant paradigm for examining perceptual sensitivity in the literature on spatial attention (Carrasco, PenpeciTalgar, \& Eckstein, 2000; Henderson, 1996; Hillyard, Vogel, \& Luck, 1998; Luck et al., 1996; Pestilli \& Carrasco, 2005).

One potential difficulty in attributing performance differences in cuing studies to differences in perceptual sensitivity arises from the possibility that cuing effects reflect reduction of position uncertainty in decision processes (Palmer, 1994; Shiu \& Pashler, 1994). In particular, when there is ambiguity in the location of the target (e.g., when there are multiple masked locations following target presentation), the effect of a valid cue could be to limit decision processes to the cued location, without any direct effect on perceptual sensitivity (Shiu \& Pashler, 1994). However, such position uncertainty was eliminated in the present paradigm, because there was only one target and only one masked location (Henderson, 1996; Pestilli \& Carrasco, 2005; Shiu \& Pashler, 1994). In sum, the present paradigm adopted key features of spatial attention experiments, so as to maximize the possibility that performance was driven by the effect of spatial attention on perceptual sensitivity, which allowed us to assess the modulation of spatial attention by object structure.

Finally, the paradigm was designed to ensure that effects of object structure reflected the spatial distribution of attention at the time of target presentation and could not be attributed to differences in shifts of attention within and across objects (Lamy \& Egeth, 2002). Such shifts are particularly likely to occur in paradigms that present four possible targets after the cue, one at each of the ends of the two rectangles, necessitating visual search (Hecht \& Vecera, 2007; Moore, Yantis, \& Vaughan, 1998; Shomstein \& Yantis, 2004). In the present method, there was only one target, and it was masked after only $70 \mathrm{~ms}$. Because there was only one target, there was no search component to the present method, reducing the potential contribution of attentional shifts. In addi-

1 In the standard Egly et al. (1994) paradigm, cues and targets typically have been superimposed over the object display, and it is not necessarily clear that cue and target stimuli are perceived as properties of the objects themselves (see Richard, Lee, \& Vecera 2008). 
tion, it is unlikely that participants could have executed a shift of attention to the target on invalid trials, given that the target was masked after $70 \mathrm{~ms}$ and covert shifts of attention to abruptly appearing stimuli require at least $80-100 \mathrm{~ms}$ to implement (Cheal \& Lyon, 1991; H. J. Müller \& Rabbit, 1989). Again, the goal here was to isolate the relatively early influence of object structure on the distribution of spatial attention.

\section{Experiment 1: Gradients and the Effect of Object Structure}

In Experiment 1, we examined whether spatial attention forms a sensitivity gradient over the attended object. On each trial, the target appeared at one of four possible locations relative to the cue (Figure 2A): at the cued location (valid condition), at a near location within the cued object, at a far location within the cued object, and at a far location within the uncued object. The three locations within the cued object provided an assessment of spatial gradient effects within an attended object. The far locations in the cued and uncued objects were equally distant from the cue, providing an assessment of object-based effects independently of distance. If object structure serves to shape the distribution of

A
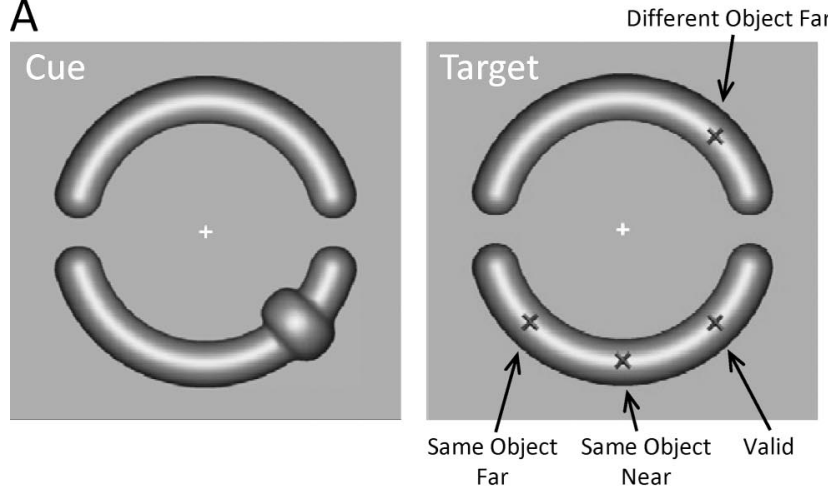

B

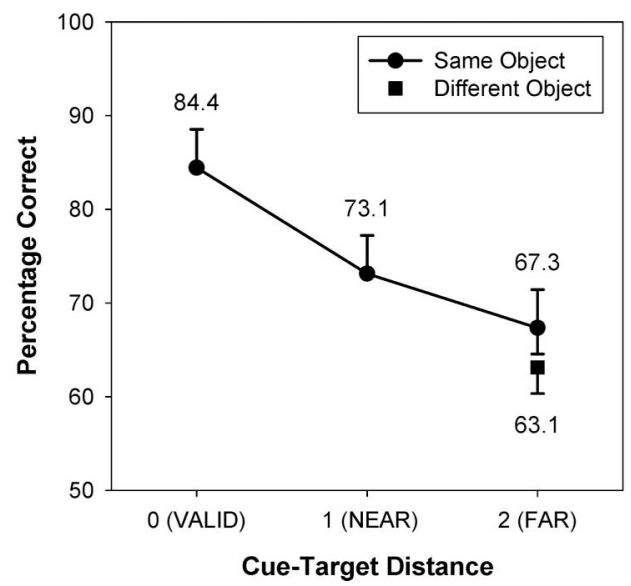

Figure 2. A, Illustration of the possible target conditions for a given cue location in Experiment 1. B, Experiment 1 mean discrimination accuracy results. Upward error bars are 95\% confidence intervals based on the error term of the within-object distance manipulation. Downward error bars are the $95 \%$ confidence interval based on the error term of the same-different object contrast at the far position. spatial attention, we should observe a gradient of perceptual sensitivity across an attended object and a same-object advantage when distance is controlled.

\section{Method}

Participants. Participants in all experiments were recruited from the University of Iowa community and were between the ages of 18 and 30. Participants reported normal or corrected-tonormal vision. They either received course credit or were paid. Ten participants completed Experiment 1.

Stimuli and apparatus. The tube objects were rendered from 3D models (see Figures 1 and 2). Because the tubes were semicircular, all cue and target locations on the tubes were equally distant from fixation. The tube objects were blue, presented against a gray background, with a central, white fixation cross $\left(0.6^{\circ} \times\right.$ $0.6^{\circ}$ ). The two tubes together subtended $13.6^{\circ}$. Tube width was $2.1^{\circ}$, and the gap between tubes was $1.2^{\circ}$ at its smallest point. Two tube orientations were possible; the gaps appeared either on the horizontal or vertical axis.

There were four possible cue locations, each near one end of a tube. Each of the four cue locations was offset $45^{\circ}$ from the horizontal and vertical axes (i.e., at the corners of a virtual square centered at fixation). Cues and targets were $5.9^{\circ}$ from fixation (measured to the center). The cue animation consisted of five frames $(10 \mathrm{~ms}$ each). In the first three frames, a bulge grew systematically out of the object. The bulge region had a width of $2.5^{\circ}$ in the first frame, $2.75^{\circ}$ in the second frame, and $3.0^{\circ}$ in the third frame. In frames 4 and 5, the bulge contracted back into the object and was no longer visible after the fifth frame.

The discrimination targets were an ' $\mathrm{X}$ ' $\left(0.8^{\circ} \times 0.8^{\circ}\right)$ and an ' $\mathrm{O}$ ' $\left(1.0^{\circ} \times 1.0^{\circ}\right)$. The mask was constructed by superimposing the ' $\mathrm{X}$ ' and 'O,' which completely obscured the contours of the targets. The target appeared at one of four possible locations relative to the cue (see Figure 2A): at the cued location (valid), at a near location within the cued object (same object near), at a far location within the cued object (same object far), or at a far location within the uncued object (different object far). When the target appeared at the cued location, it was presented at the center of the cued region. The two far locations had an angular offset of $90^{\circ}$ from the cue location, around the circumference of the tube display. They were therefore equated for absolute distance from the cue. The near location was offset $45^{\circ}$ from the cue location, along the cued object. The Euclidean distance from the center of the cue location to the center of the target location was $8.4^{\circ}$ for the two far locations and $4.6^{\circ}$ for the near location.

Stimuli were displayed on a 17 -in CRT monitor with a $100-\mathrm{Hz}$ refresh rate. A forehead rest was used to maintain a constant viewing distance of $80 \mathrm{~cm}$. Responses were collected using a serial button box with millisecond accuracy. The experiment was controlled by a PC running E-Prime software (Schneider, Eschmann, $\&$ Zuccolotto, 2002). All images for each trial were preloaded into graphics memory before the trial commenced. The presentation of each stimulus was synchronized with the monitor's vertical retrace. The $100-\mathrm{Hz}$ refresh rate allowed stimulus durations in 10-ms increments.

Procedure. On each trial, participants first pressed a button to initiate the trial. There was a blank delay (fixation cross only) of $500 \mathrm{~ms}$. This was followed by the events depicted in Figure 1. The 
two tube objects were displayed for $1000 \mathrm{~ms}$, followed by the cue animation (three frames of bulge growth and 2 of recession over 50 ms). The cue animation was followed by a 70-ms ISI. Then, the discrimination target was presented for $70 \mathrm{~ms}$, followed by the mask until response.

Participants rested the index and middle finger of the right hand on two buttons of the serial button box. They pressed the left button to indicate an ' $\mathrm{X}$ ' target and the right button to indicate an ' $\mathrm{O}$ ' target. Because the measure of interest was discrimination accuracy, participants were instructed to respond as accurately as possible and to take as long to respond as was necessary (but no longer). Incorrect responses were followed by the word "incorrect" presented in the center of the screen for $300 \mathrm{~ms}$. There was a 600-ms delay between trials.

In addition to the instructions described above, participants were instructed to fixate the central cross and to keep their eyes fixed at that location throughout the trial. Even with this instruction, it is important to ensure that eye movements could not have influenced performance. In particular, we must ensure that participants could not have reliably executed a saccade to the cued location before the masking of the target. To assess the speed with which a saccade could be directed to the cued region of the tube object, we examined data from a control experiment $(n=8)$. In this experiment, participants were required to execute a saccade as quickly as possible to the cued region of the tube object. That is, the tube stimuli and "bulge" cue were identical to those used in Experiment 1 , except that instead of maintaining central fixation, participants executed a saccade to the cued region. We measured the elapsed time from the start of the cue to the beginning of the first fixation on the cued region. Mean elapsed time was $245 \mathrm{~ms}$. In Experiment 1 of the present study, the SOA between the beginning of the cue and the mask was $190 \mathrm{~ms}$. Therefore, if participants had executed a saccade to the cued region in Experiment 1, their next fixation typically would have begun $55 \mathrm{~ms}$ after the target had been masked. We also examined the very fastest elapsed times in the control experiment, which were in the range of 170-190 ms. On these trials, the eyes would have landed $10-20 \mathrm{~ms}$ before the onset of the mask, but these trials constituted only $9 \%$ of the total trials, and it is unlikely that any useful target information could have been extracted in the 10-20 ms before the mask. In sum, participants could not have reliably completed a saccade to the cued region before the onset of the mask. As a result, saccades would have greatly impaired performance of the target discrimination task, and thus it is unlikely that participants generated them (or at least persisted for any length of time in generating them).

After arriving at the lab, participants first provided informed consent and received instructions. They completed two experiment blocks, one in which the gap between the tube objects was on the vertical axis and the other in which the gap was on the horizontal axis. Tube orientation was blocked so that perceptual aftereffects from the previous trial would not influence perception of the two objects, a possibility if trials with the two tube orientations were randomly mixed. However, subsequent experiments suggest that this precaution was probably not necessary. Block order was counterbalanced across participants. Each block began with 12 practice trials, drawn randomly from the full design. Practice was followed by 320 experiment trials: $224(70 \%)$ in the valid condition and $32(10 \%)$ in each of the three invalid conditions (same object near, same object far, different object far). The trials were divided evenly among the four cue locations and the two targets. Participants completed a total of 640 experiment trials. The entire session lasted approximately $50 \mathrm{~min}$.

\section{Results}

To minimize possible floor effects, we set a criterion for participant inclusion of at least $60 \%$ correct overall on the target discrimination task (chance $=50 \%$ ). Two participants fell below this criterion and were replaced.

We examined percent correct performance on the target discrimination task as a function of cue validity, same-different object, and distance. Accuracy data appear in Figure 2B. ${ }^{2}$

Consistent with the grouped array claim that attention forms a spatial gradient across the attended object, there was a reliable effect of distance within the cued object, with discrimination accuracy decreasing with increasing cue-target distance, $F(2$, $18)=20.0, p<.001$. This effect was quite remarkably large in absolute terms. Discrimination accuracy was fully $17 \%$ higher when the target appeared at the cued location than when it appeared at the far location within the cued object.

Consistent with the grouped array claim that object selection results in differences in perceptual sensitivity, there was also a reliable effect of same-different object. Discrimination accuracy in the same object far condition $(67.4 \%)$ was reliably higher than accuracy in the different object far condition $(63.1 \%), F(1,9)=$ $5.85, p=.039$, which were equated for absolute distance from the cued location.

\section{Discussion}

In Experiment 1, perceptual discrimination performance declined smoothly as a function of the within-object distance between the cue and the target. Attention directed to an object appears to form a spatial gradient across the object. This finding is consistent with 1) the claim that attention to objects involves the selection of the object by an inherently spatial mechanism, and 2) evidence that the distribution of spatial attention is graded. In addition, when distance was controlled we observed a discrimination advantage when the target appeared in the same object as the cue, replicating the basic finding of Egly et al. (1994). The present paradigm was optimized to measure the effects of object structure on perceptual sensitivity. Thus, the same-object advantage indicates that attention to objects involves selection by a mechanism that directly influences perceptual sensitivity, again consistent with the claim that object structure serves to modulate the allocation of spatial attention.

We have, at this point, demonstrated both gradient effects and object effects using the masked discrimination paradigm. However, given that this method diverges in a number of significant ways from the original Egly et al. (1994) paradigm, we sought to ensure that we would observe the same pattern of results using an

\footnotetext{
2 Although reaction time data are not directly relevant for interpreting unspeeded, masked discrimination experiments, for completeness, reaction time data in each of the masked discrimination experiments are reported in the Appendix. In each experiment, the pattern of reaction time data either complemented the accuracy data or was neutral with regard to the accuracy data.
} 
onset detection paradigm similar to the original Egly et al. experiments. The basic method of this follow-up experiment is illustrated in Figure 3A. The key difference between the onset detection and masked discrimination methods was the target stimulus. In this onset-detection follow-up experiment, the target was a small dot that grew out of the tube object over three 10-ms frames of animation. It remained visible until response. The onset dot appeared either at the cued location or at one of the three invalid locations. Catch trials were included on which no dot appeared. Participants were instructed to respond as quickly as possible when a dot appeared and to withhold response if no dot appeared. Reaction time was measured from the beginning of the dot onset animation. If no response was registered within $2000 \mathrm{~ms}$ after the dot onset, the computer terminated the trial.

In this onset detection version of Experiment $1(n=10)$, participants completed 768 experiment trials: $448(58.3 \%)$ in the valid condition, $64(8.3 \%)$ in each of the three invalid conditions (same object near, same object far, different object far), and 128 catch trials $(16.7 \%)$ in which no target appeared. Participants pressed the response button within $2000 \mathrm{~ms}$ on every trial that contained a target dot (i.e., there were no misses). To ensure that RT data were not contaminated by anticipatory responses, we set a criterion of $85 \%$ correct on catch trials. One participant fell below this criterion and was replaced. RTs below $100 \mathrm{~ms}$ or above $600 \mathrm{~ms}$ were removed as outliers ( $4.1 \%$ of data). The trimming of RT outliers did not alter the pattern of data.

RT results are reported in Figure 3B. We replicated the principal findings of Experiment 1. First, there was a spatial gradient effect within the cued object. Onset-detection RT increased as the distance between the cue and the target increased, $F(2,18)=7.79$, $p=.004$. Second, we observed an effect of same-different object, with shorter mean RT in the same object far condition than in the different object far condition, $F(1,9)=6.38, p=.032$, replicating Egly et al. (1994). ${ }^{3}$

In both the masked discrimination and onset detection methods, we observed a spatial gradient of attention across the cued object and a same-object advantage when distance was controlled. These results are consistent with the grouped array theory, holding that object structure modulates the distribution of spatial attention. However, two alternative accounts must be eliminated. First, it is possible that the gradient effects in Experiment 1 were caused by the fact that far targets were closer to the edge of the objects than near targets; cue-target distance was confounded with the position of the target within the object. This issue was addressed in Experiment 2. Second, it is possible that the results of Experiment 1 were generated by the concatenation of two effects: 1) a purely objectbased effect caused by having to divide or switch attention between object representations (independently of spatial position), and 2) a purely spatial effect, generating the spatial gradient independently of object structure. ${ }^{4}$ If so, then the present results would not necessarily support our hypothesis that the object and gradient effects were generated by an interaction between object structure and the distribution of spatial attention. These issues were addressed in Experiments 3 and 4.

\section{Experiment 2: The Spatial Gradient Within an Object}

In Experiment 2, we examined the effect of cue-target distance using a single "ring" object (Figure 4A). One of eight possible locations was cued, followed by the discrimination target stimulus at the cued location or at one of the remaining seven locations. The distance between cue and target was either $0,1,2,3$, or 4 target positions. Each location was equally likely to be cued, and the target appeared at each location an equal number of times in each distance condition.

The use of a ring supported a larger range of within-object cue-target distances than used in Experiment 1, allowing us to map more comprehensively the spatial distribution of attention across the object. In addition, effects of distance from the cue could not be attributed to proximity to the ends of the object (a limitation of the design of Experiment 1), because the object was continuous.

\section{Method}

Participants. Ten new participants completed Experiment 2.

Stimuli and apparatus. The tube objects, cues, and targets were identical to the stimuli in Experiment 1, with the following exceptions. Instead of two semicircular tube objects, a single tubular ring was presented. There were eight possible cue locations, evenly spaced along the ring (separated by an angular offset of $45^{\circ}$ ). The target could appear at the cued location (valid) or at any one of the seven uncued locations. The distance from cue to the target was either 0 (valid), 1, 2, 3, or 4 object positions, allowing us to map the gradient of attention across a larger spatial extent than in Experiment 1.

The apparatus was the same as in Experiment 1.

Procedure. The events in a trial were the same as in Experiment 1.

Participants first completed a practice session of 24 trials, drawn randomly from the full design. Practice was followed by a single experiment session of 672 experiment trials: $336(50 \%)$ in the valid condition and $336(50 \%)$ divided evenly among the seven invalid locations (48 each). The trials were divided evenly among the eight cue locations and the two targets. The entire session lasted approximately $50 \mathrm{~min}$.

\section{Results and Discussion}

Accuracy data are reported in Figure 4B. Replicating Experiment 1 , there was a robust spatial gradient within the object. Discrimination accuracy decreased significantly with increasing cue-target distance, $F(4,36)=21.7, p<.001$. The gradient reached floor by three object positions from the cue, suggesting that attentional enhancement was limited to a relatively local region near the cued location.

We also conducted an onset detection version of Experiment 2 $(n=10)$. Participants completed 704 experiment trials, $352(50 \%)$

\footnotetext{
3 We also replicated the Egly et al. (1994) object effect in additional onset-detection experiment $(n=10)$ that used only the three target conditions probed by Egly et al. (valid, $306 \mathrm{~ms}<$ invalid same object, 332 $\mathrm{ms}<$ invalid different object, $342 \mathrm{~ms}$ ). All pairwise differences were statistically reliable at the .05 level.

4 The former is not particularly plausible, given that the $70 \mathrm{~ms}$ presentation of the target was likely to preclude a shift of attention from the cue to an invalid target before it was masked. However, we cannot rule out an explanation in terms of attention shifts on the basis of timing alone, as we do not have direct evidence of the speed of covert attention shifts within this paradigm.
} 

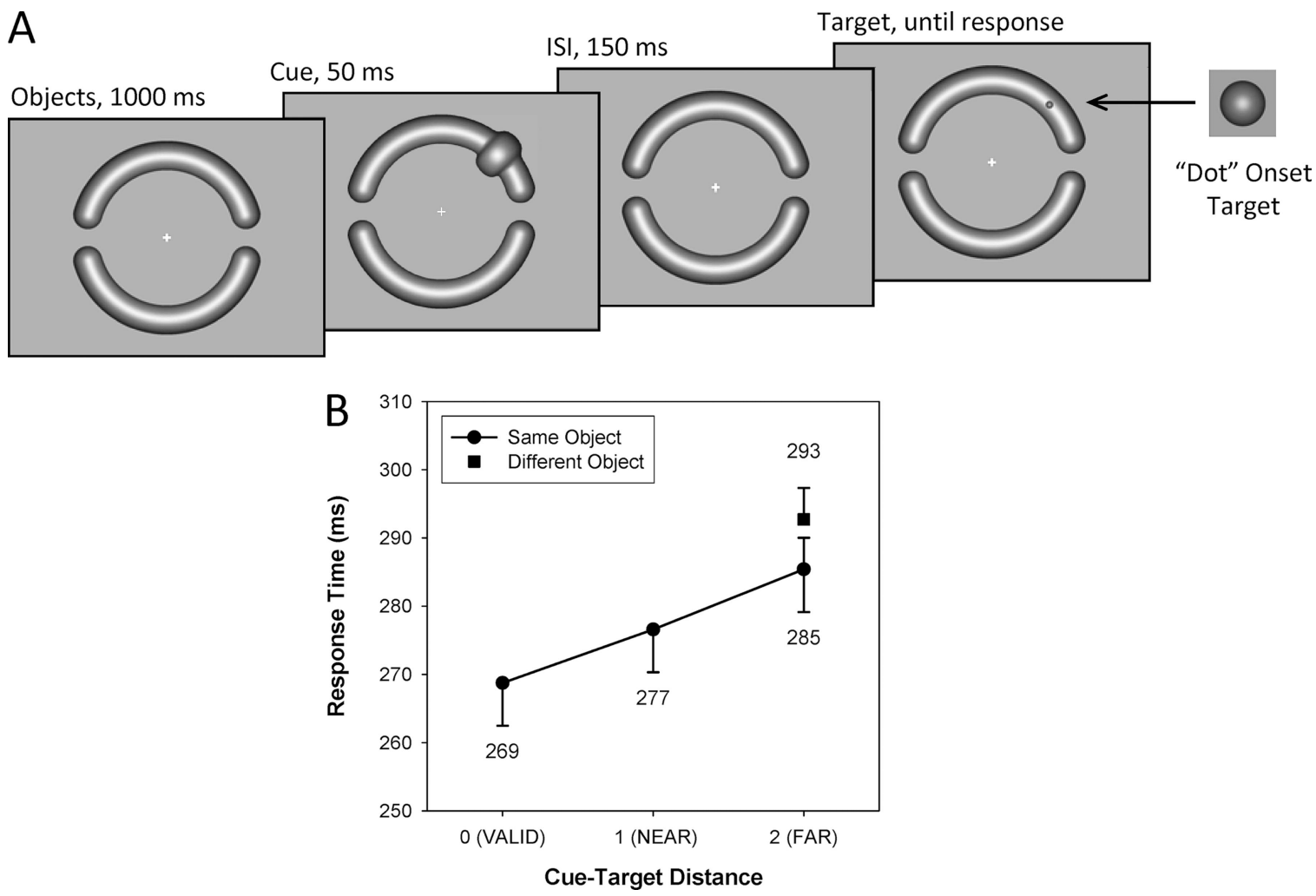

Figure 3. A, Sequence of events in a trial of the onset detection follow-up to Experiment 1. B, Mean RT for the onset detection follow-up experiment. Downward error bars are $95 \%$ confidence intervals based on the error term of the within-object distance manipulation. Upward error bars are the $95 \%$ confidence interval based on the error term of the same-different object contrast at the far position.

in the valid condition, $224(31.8 \%)$ divided evenly between the seven invalid locations, and 128 catch trials (18.2\%). Participants pressed the response button on all but six trials that contained a target dot $(99.9 \%)$. One participant fell below the $85 \%$ correct catch trial criterion and was replaced. RT trimming eliminated $3.8 \%$ of the data.

Mean RT results are reported in Figure 4C. Replicating Experiment 2 , there was a reliable effect of cue-target distance, with RT increasing as cue-target distance increased, $F(4,36)=9.9, p<$ .001. As in Experiment 2, the gradient of attention was limited to a relatively local region of the object.

In both versions of the task, a robust spatial gradient was observed across the object, consistent with the assumptions of the grouped array model. Interestingly, the region of facilitation within the object was limited to target positions relatively near the cue. Note, however, that the object stimulus used here was quite large $\left(13.6^{\circ}\right.$ across). So, although the region of facilitation was small relative to the area of the entire object, it extended at least $8.4^{\circ}$ in space (the distance between the two target locations lying on each side of the cued location). Facilitation limited to locations near the cue could also be attributable to the fact that participants had strong incentive to keep attention focused at the cued region of the object. Without such a task demand, attention might have been more uniformly distributed within the object.

\section{Experiment 3: The Role of Object Boundaries in Selection}

In experiments using the Egly et al. (1994) paradigm, a standard explanation of the object advantage (i.e., better target perception for within-object invalid trials compared with between-object invalid trials) is that the effect arises as a result of costs associated with dividing or switching attention between object representations. The grouped array account holds that the within-object advantage can arise from a different cause: The boundaries of the attended object constrain the spatial distribution of attention (Avrahami, 1999; Marino \& Scholl, 2005), causing attention to be limited to or concentrated within the cued object, with consequent effects on the efficiency of the perceptual processing of the target. As discussed in the Introduction, these two broad forms of objectbased selection need not be mutually exclusive. However, in the grouped-array account, the presence of an object boundary is a critical feature generating the object-based effect. If such a boundary could be created within a single object, the spread of attention would be similarly constrained by the boundary, generating an object-based effect within a single object. Unlike the object effect in the original Egly et al. task (or in Experiment 1), an object effect of this type could not be attributed to a demand to divide or switch attention between discrete object representations, because there would be only a single object. Moreover, a one-object condition 
removes any relationship between the cued object and the target location; targets would be no more likely to appear in the cued object than in an uncued object, because all target locations would be within the single, cued object.

To create this type of test, we used the tube stimuli from Experiment 1, but introduced only a single gap in the tube (see Figure 5A). This generated a single object stimulus with a salient internal discontinuity. Across trials, the gap could appear at one of

A
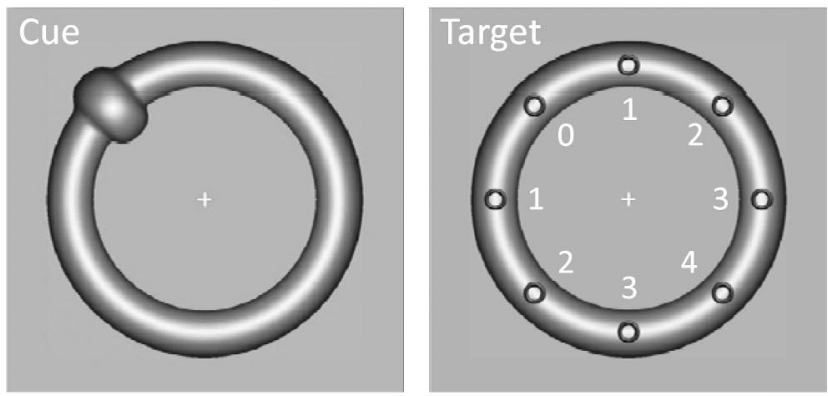

B

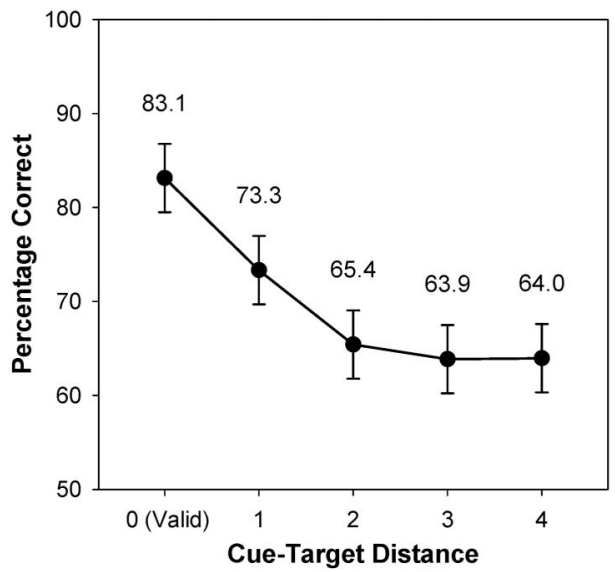

C

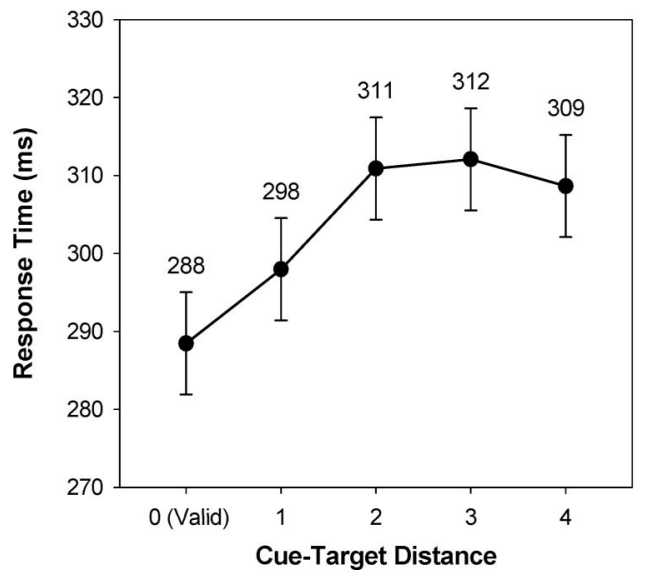

Figure 4. A, Illustration of the possible target conditions for a given cue location in Experiment 2. B, Experiment 2 discrimination accuracy results. Error bars are $95 \%$ confidence intervals based on the error term of the cue-target distance manipulation. C, Mean RT for the onset detection follow-up to Experiment 2. Error bars are 95\% confidence intervals based on the error term of the cue-target distance manipulation.

\section{A. One Object Condition}
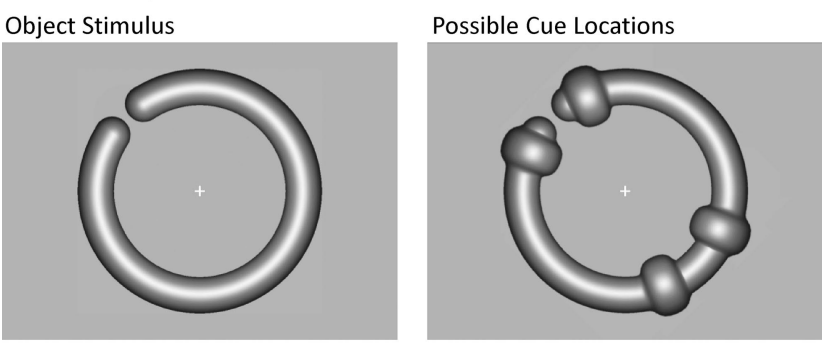

\section{B. Two Object Condition}

Object Stimulus

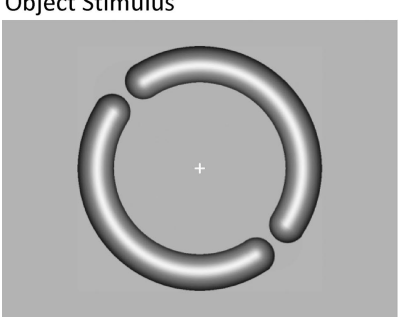

Possible Cue Locations

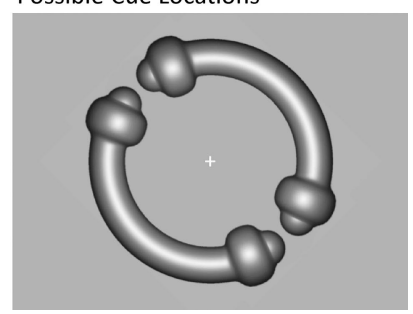

Figure 5. Possible cue locations for a particular gap location in Experiment 2 in the one-object condition (A) and in the two-object condition (B).

eight positions around the tube. For each tube stimulus, the cue appeared in one of four possible locations relative to the gap (Figure 5A). Two cue locations were near the gap, and two were at the opposite positions in the middle of the object. The cue locations near the gap were of primary interest, as they allowed mapping of the spatial gradient across the gap. (The cue locations in the middle of the object ensured that participants would not strategically attend to the gap region prior to the cue.) Figure $6 \mathrm{~A}$ shows the three possible target locations for a given cue appearing near a gap. The two invalid locations are equidistant from the cued location. However one lies across the gap from the cue (invalid across gap), and the other lies along the continuous extent of the object (invalid along object). According to the account developed here, spatial attention directed to the cue should spread along the continuous extent of the object, and the invalid-along-object target should benefit from this gradient of attention (as found in Experiments 1 and 2). However, the spread of attention in the direction of the gap should be blocked or retarded by the contours of the gap. Attention should not spread as efficiently to the invalid-across-gap target, and perception of that target should be impaired relative to the invalid-along-object target. ${ }^{5}$

In addition, to assess the magnitude of the object-based effect caused by a gap in a single object, the one-object condition was compared with the standard two-object condition, which was identical to the one-object condition except for the presence of a second gap (Figures 5B and 6B).

\footnotetext{
5 Attention could, in theory, spread all the way around the continuous extent of the object until it reached the across-gap location. However, Experiment 2 showed that the gradient of attention is limited to a local region of the object and would not extend far enough for this to be a concern.
} 


\section{A. One Object Condition}
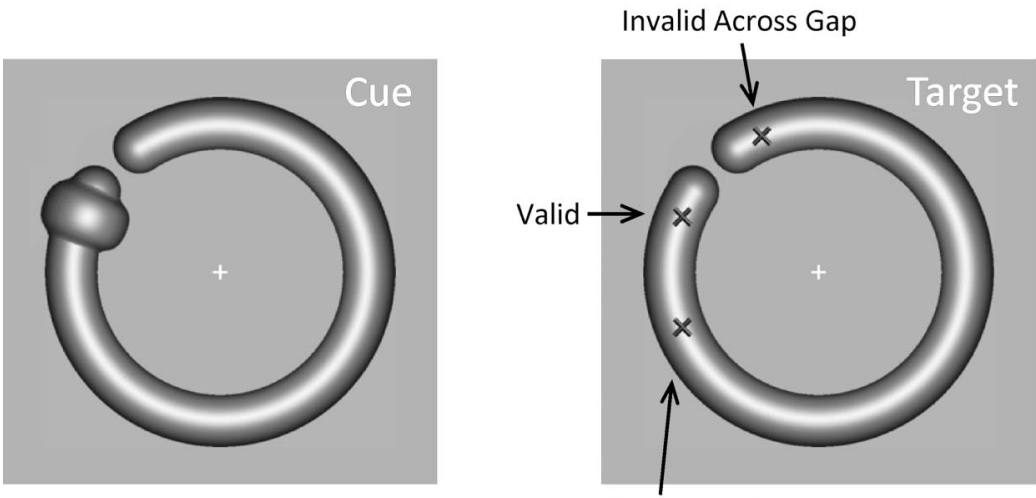

Invalid Along Object

\section{B. Two Object Condition}
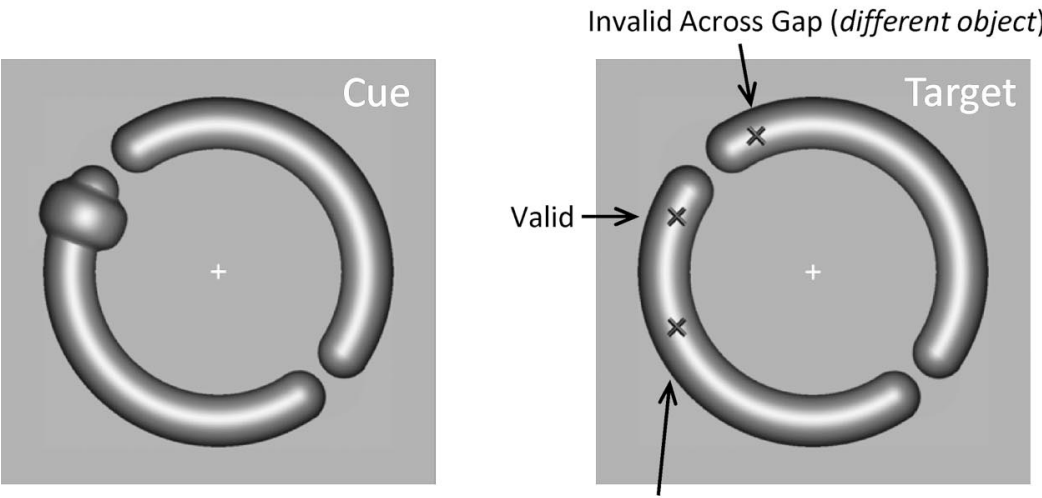

Invalid Along Object

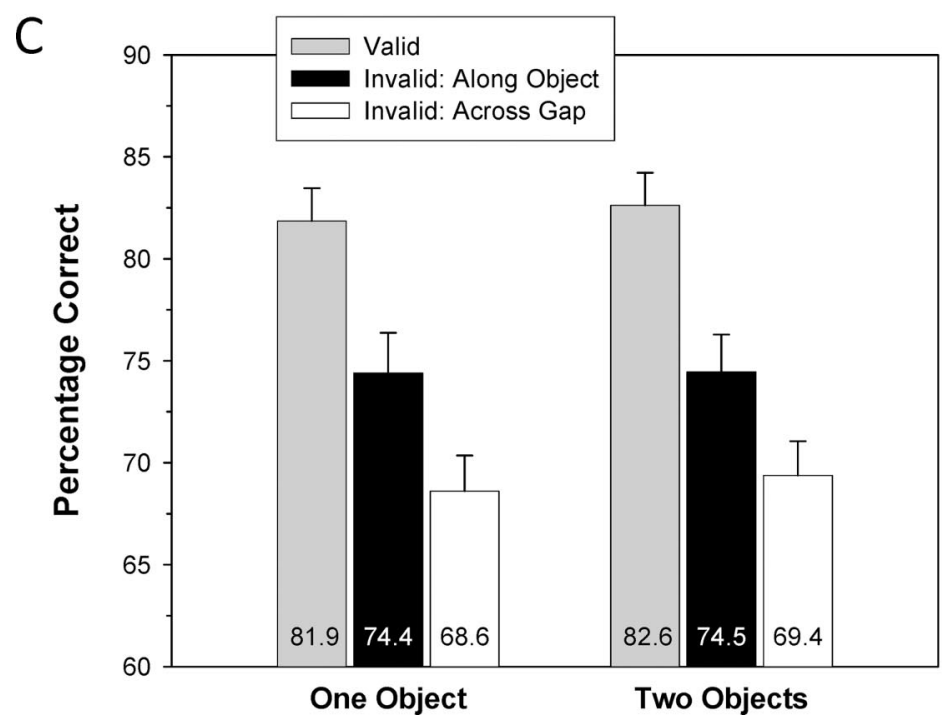

Figure 6. A, Illustration of the possible target conditions for a given cue location in the one-object condition of Experiment 3. B, Illustration of the possible target conditions for a given cue location in the two-object condition of Experiment 3. C, Mean discrimination accuracy results in Experiment 3. 


\section{Method}

Participants. Forty new participants completed Experiment 3.

Stimuli and apparatus. In the one-object condition, there was a single gap in the tube. The size of the gap was $0.46^{\circ}$ at its smallest point. The gap could appear at one of eight locations on the tube, evenly spaced around the ring (separated by an angular offset of $45^{\circ}$ ). In the two-object condition, two gaps were present, separated by $180^{\circ}$.

The cue could appear in one of four possible locations relative to the gap(s), as illustrated in Figure 5. In the one-object condition, two of the possible cue locations were near the gap (each offset $22.5^{\circ}$ from the gap location). The other two possible locations appeared in the middle of the object, opposite the locations near the gap. The cues in the middle of the object were included so that participants would not have an incentive to preattend to the gap region (these trials were excluded from the analyses examining the effect of gap). In the two-object condition, the possible cue locations were the same, but given the presence of a second gap, all cues were near a gap.

The target could appear in three possible locations relative to the cue and relative to the gap (see Figure 6). In the valid condition, the target appeared at the cued location. In the two invalid conditions, the target was presented at a location offset $45^{\circ}$ either clockwise or counterclockwise from the cued location (the same distance from the cue as in the "near" condition of Experiment 1). In the along-object condition, the target appeared along the object in the direction opposite to the gap. In this condition, the target appeared within the cued object, regardless of whether there was one or two objects. In the across-gap condition, the target appeared across the gap from the cue. In this condition, when there was one object, the target still appeared in the same object as the cue. However, when there were two objects, the target appeared in a different object than the cue. The Euclidean distance between the cue and target in the two invalid conditions was $4.6^{\circ}$.

The apparatus was the same as in Experiment 1.

Procedure. The events in a trial were the same as in Experiment 1.

Participants first completed a practice session of 24 trials, drawn randomly from the full design. Practice was followed by a single experiment session of 768 experiment trials: $512(67 \%)$ in the valid condition and 256 (33\%) divided evenly between the two invalid conditions. The trials were divided evenly among the two object configurations (one object or two objects), the eight gap locations, the four cue locations, and the two targets. Thus, across the experiment, each of the absolute locations around the ring was equally likely to be cued and was equally likely to be the target location. The entire session lasted approximately $55 \mathrm{~min}$.

Thirty of the participants completed the procedure described above. Given the importance of the one versus two object manipulation, we sought to ensure that participants did indeed perceive the appropriate number of objects (one or two). To do so, the task was modified slightly for the remaining 10 participants. For these participants, when the object(s) appeared at the beginning of the trial, the participant reported (by button press) whether the display contained one or two objects. After this response, the trial proceeded as described above. Accuracy for reporting the number of objects was $99.1 \%$ correct, demonstrating that participants easily discriminated the one and two object displays. The $0.9 \%$ of trials on which the participant incorrectly reported the number of objects was eliminated from analysis. The data from the two groups of participants produced precisely the same pattern of results: There was no main effect of group nor any interaction between group and other variables. Thus, the data from the two groups were combined in the main analyses, reported below.

\section{Results}

The main analyses were conducted over data from cue locations near a gap (see Figure 6), which allowed us to probe the effect of object discontinuity on the distribution of attention. For the twoobject condition, data from all four cue locations were included, as all cues locations were near a gap. For the one-object condition, only the data from the two cue locations near the gap were included.

Mean accuracy data are reported in Figure 6C. We first entered the data into a 2 (one object, two objects) $\times 3$ (cue validity condition) ANOVA. There was a main effect of cue validity, $F(2$, $78)=83.0, p<.001$, but no effect of the number of objects, $F<$ 1 , and no interaction between these factors, $F<1$.

Of central importance, there was a reliable effect of gap in both the one-object condition and the two-object condition. In the one-object condition, accuracy in the across-gap condition was $5.80 \%$ lower than accuracy in the along-object condition, $F(1$, $39)=12.2, p<.001$. An effect of gap was observed despite the fact that there was only one object visible. Thus, object discontinuities are capable of generating an object-based effect of attention without any demand at all to divide or shift attention between discrete object representations. In the two-object condition, accuracy in the across-gap condition was $5.08 \%$ lower than accuracy along the object, $F(1,39)=12.2, p<.001$. There was no interaction between number of objects and along object/across gap, $F<1$. The magnitude of the gap effect did not differ between the one-object and two-object conditions, suggesting that a local object discontinuity (gap) in a single object is just as effective in generating object-based effects as the standard two-object stimulus (at least in the present method).

A possible concern with Experiment 3 is that the target location was closer to the end of the object in the across-gap condition than in the along-object condition. To ensure that differences in lateral masking did not contribute to the performance difference between these conditions, we compared valid-trial performance when the cue and target appeared near the end of the object versus when the cue and target appeared in the middle of the object in the oneobject condition (see Figure 5A). Valid trial performance when the cue and target appeared near the end of the object was $82.6 \%$ correct in the two-object condition and $81.9 \%$ correct in the one-object condition. Valid trial performance when the cue and target appeared in the middle of the object in the one-object condition was $81.1 \%$ correct. Target discrimination was actually slightly better for target locations near the end of an object than for target locations in the middle of an object, and lateral masking therefore was unlikely to have contributed to lower performance at the across-gap location compared with the along-object location.

To be perfectly sure that lateral masking did not contribute to the gap effect, we conducted a further control experiment $(n=12)$. A one-object stimulus (with a single gap) was presented on every trial (See Figure 5A). As in Experiment 3, the gap was equally 
likely to appear at each of eight locations around the ring (separated by $45^{\circ}$ ), and the target locations nearest the gap were offset from the gap by $22.5^{\circ}$. Participants first saw the object stimulus for $1000 \mathrm{~ms}$. This was followed by the target and mask. There was no cue. On each trial, the target was equally likely to appear at one of eight target locations spaced evenly around the ring (separated by $45^{\circ}$ ). This method provided a baseline measure of target perceptibility at different positions relative to the gap. Discrimination performance for targets nearest to the gap $(75.4 \%)$ was essentially the same as discrimination performance for the other target locations within the object $(75.5 \%), F<1$. Again, there was no evidence of lateral masking of targets near the gap.

\section{Discussion}

In Experiment 3, a gap in a single object constrained the spread of attention within that object, generating a decrement in target discrimination when an invalid target appeared across a gap from the cue compared with when an equidistant invalid target appeared along the continuous extent of the object. The magnitude of this effect was no smaller than the decrement generated when an invalid target appeared in a different object than the cue. The results of the one-object condition cannot have been caused by having to divide or switch attention between discrete object representations, because there was only one object. In addition, the results cannot have been a result of the strategic prioritization of within-object locations (Shomstein \& Yantis, 2002); with only one object, all target locations were within the cued object. Therefore, the present results provide strong support for the grouped array claim that object perceptual structure (particularly object boundaries) shapes the spatial distribution of attention, blocking or retarding the spread of attention across the boundaries of the object.

\section{Experiment 4: Mapping the Spatial Gradient Across an Object Boundary}

Thus far, we have observed gradient effects within an attended object, and we have demonstrated that object boundaries (even boundaries within a single object) serve to constrain the distribution of attention. It still remains a possibility, however, that the gradient effects observed in Experiments 1 and 2 were generated independently of object structure. That is, the graded discrimination performance could reflect the graded allocation of spatial attention without regard to the structure of the object. To test directly whether the gradient of spatial attention interacts with object structure, in Experiment 4 we mapped the gradient of attention across a gap in a single object and compared it with the gradient formed along the continuous extent of the object. If the gradient effects in previous experiments were generated by a spatial mechanism that is not sensitive to object structure, the spatial gradient should not be sensitive to the gap in Experiment 4. However, if an object boundary blocks or retards the spread of attention, as held by the grouped array theory, the attentional gradient should be eliminated or reduced across a gap in an object. Whether an object boundary completely blocks or simply retards the spread of attention is an open question. Recently, Kravitz and Behrmann (2008) found evidence for a spatial gradient formed outside the boundaries of the object (see also Cole, Gellatly, \&
Blurton, 2001), which they argued resulted from the propagation of the within-object activation to nearby, object-external regions with overlapping receptive fields. On the basis of the Kravitz and Behrmann results, we expected that object boundaries in Experiment 4 would not completely block the spread of attention across the gap and that we would observe a diminution, but not an elimination, of the spatial gradient across the gap.

The one-object stimuli from Experiment 3 were used (Figure 7A). To map the gradient along the object and across the gap, we used a larger number of target locations (8) spaced evenly around the entire object (as in Experiment 2).

\section{Method}

Participants. Twenty-five new participants completed Experiment 3 .

Stimuli and apparatus. The stimuli were identical to the one-object condition of Experiment 3. The cue could appear in one of four possible locations relative to the gap. Two of the possible cue locations were near the gap (each offset $22.5^{\circ}$ from the gap location). The other two possible cue locations appeared in the middle of the object, opposite the locations near the gap. Again, the cues in the middle of the object were included only so that participants would have no incentive to preattend to the gap region, and these trials were excluded from the analyses.

The target could appear in eight possible locations relative to the cue and relative to the gap (see Figure 7A). In the valid condition, the target appeared at the cued location. In the seven invalid conditions, the target was presented in one of the remaining seven target locations. The distance between the cue and target was either $0,1,2,3$, or 4 object positions. These locations either lay along the object or across the gap (although this designation becomes ambiguous for the locations that were furthest from the gap).

The apparatus was the same as in Experiment 1.

Procedure. The events in a trial were the same as in Experiment 1.

Participants first completed a practice session of 24 trials, drawn randomly from the full design. Practice was followed by a single experiment session of 704 experiment trials: 256 (36.4\%) in the valid condition and 448 (63.6\%) divided evenly between the seven invalid locations. Trials were divided evenly between the eight gap locations, four cue positions, and two targets. The entire session lasted approximately $45 \mathrm{~min}$.

\section{Results and Discussion}

Because we were interested in the modulation of the spatial gradient of attention by object boundaries, five participants (of 25) were replaced because they did not show a baseline cuing effect, perhaps because valid cues constituted a smaller proportion of trials in this experiment than in earlier experiments (in which every single subject showed a baseline cuing effect). The replacement of these participants did not change the pattern of results.

Of central interest was performance on trials when the cue appeared near the gap. The target could either appear at the cued location (valid), at an uncued location across the gap, or at an uncued location further along the object. Accuracy data for acrossgap and along-object targets are presented in Figure 7B. For the along-object condition, there was a reliable effect of distance for 

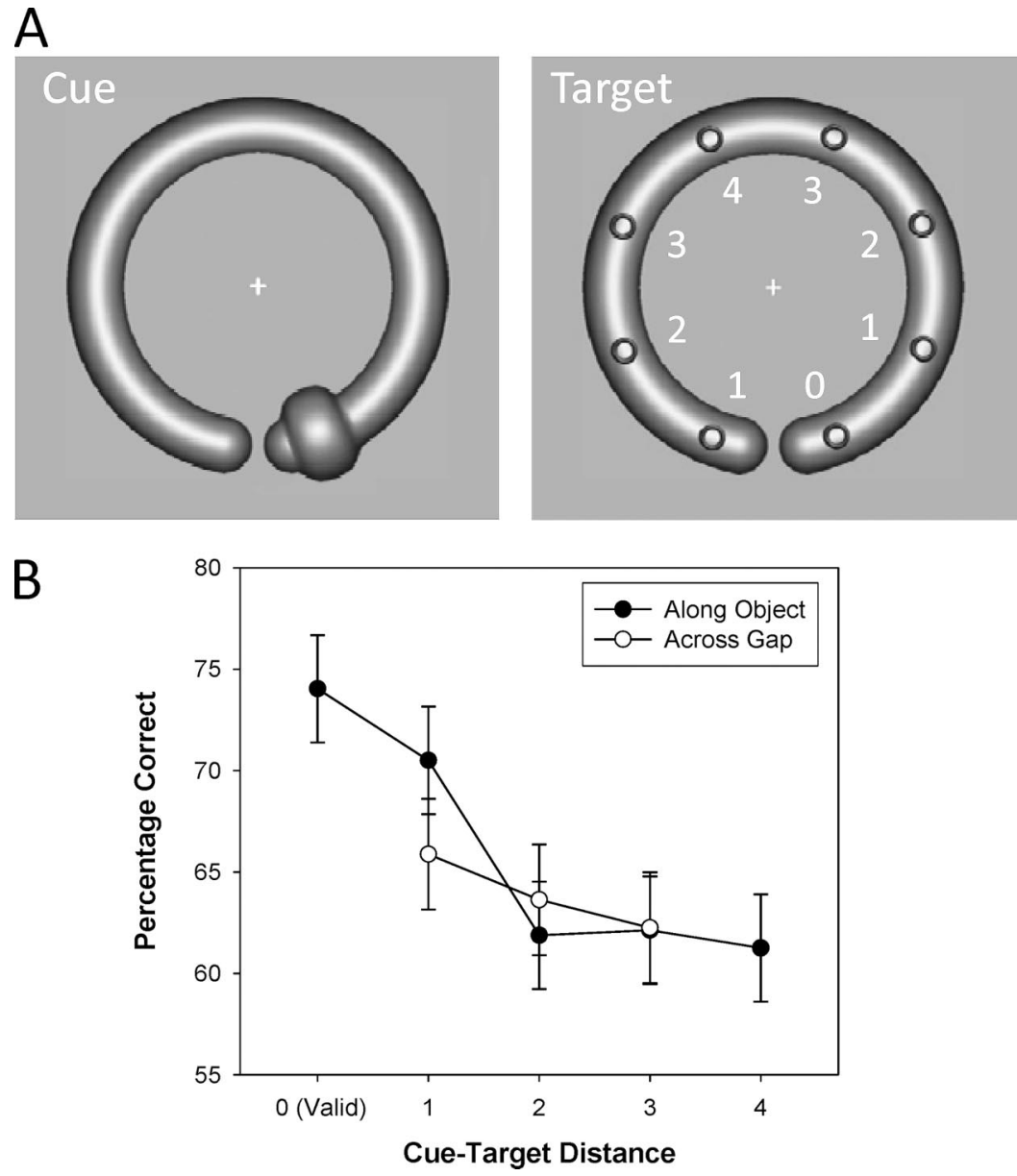

Figure 7. A, Illustration of the possible target conditions for a given cue location in Experiment 4. B, Mean discrimination accuracy results in Experiment 4.

target positions 1,2 , and $3, F(2,48)=11.6, p<.001$. For positions 1,2, and 3 across the gap, there was not a reliable effect of distance, $F(2,48)=1.81, p=.174$, although the trend was toward lower performance with increasing distance. The interaction between gap (along object, across gap) and distance was marginally reliable for positions $1-3, F(2,48)=2.67, p=.079$, and was statistically reliable for positions 1 and $2, F(1,24)=5.31$, $p=.030$. At position 1 , discrimination accuracy was reliably higher in the along-object condition than in the across-gap condition, $F(1,24)=4.69, p=.040$, indicating diminution of attentional resources across the gap. There was no difference between gap conditions at positions 2 and $3, F \mathrm{~s}<1$.

Although there was no reliable main effect of cue-target distance for locations across the gap, accuracy at position 1 across the gap was marginally higher than accuracy at position 3 across the gap, $F(1,24)=3.49, p=.07$, and accuracy at position 1 across the gap was reliably higher than accuracy at position $4, F(1,24)=$ $5.86, p=.02$. Thus, the data are at least consistent with some degree of attentional spread, and a weak gradient, across the gap.

As in Experiment 3, position 1 was closer to the end of the object in the across-gap condition than in the along-object condition. To assess possible lateral masking at the end of the object, we again compared valid-trial performance when the cue and target appeared near the end of the object versus in the middle of the object. Discrimination performance at the ends of the object was actually numerically higher $(74.0 \%)$ than in the middle of the object $(73.1 \%), F<1$.

In summary, the spatial gradient of attention was significantly attenuated across the gap. The spatial allocation of attention is clearly modulated by object structure. As in Experiment 3, this constitutes an object-based effect of attention with only one object, which could not have been caused by the need to divide or switch attention between two discrete object representations. Interestingly, the object boundaries did not appear to completely block the spread of attention across the gap, consistent with the recent finding of gradient effects in the areas surrounding an object (Kravitz \& Behrmann, 2008).

\section{General Discussion}

In the present study we examined the means by which object structure modulates the spatial distribution of attention. Under grouped array accounts of object-based selection (Kramer \& Jacobson, 1991; Kramer, Weber, \& Watson, 1997; Vecera, 1994; 
Vecera \& Farah, 1994), the boundaries of an object are proposed to constrain the spread of spatial attention, such that attention is concentrated in regions bounded by the contours of the object. This interaction between object structure and spatial attention is proposed to occur via the top down influence of a higher-level object representation on spatially arrayed activity in relatively early visual areas. Because the grouped array theory holds that the means of selection is spatial attention, the theory predicts that attention directed to objects should exhibit hallmark properties of spatial attention. In the present study, we examined three issues central to understanding the interaction between object structure and spatial attention:

1) Does attention form a spatial gradient across an object, and what is the shape of that gradient?

2) Do object boundaries serve to constrain the spread of attention, and how do boundaries interact with the gradient of attention?

3) Does object selection influence perceptual sensitivity?

To address these questions, we developed a modified version of the Egly et al. (1994) paradigm that enabled us to probe the spatial distribution of attention across an object and the modulation of that distribution by object boundaries. In addition, the paradigm was optimized to probe the effect of object selection on perceptual sensitivity.

\section{Attention Forms a Gradient Across an Object}

Using circular and semicircular "tube" objects, we manipulated the within-object distance between the cued location on an object and the target location. A robust spatial gradient across the attended object was observed. Target discrimination performance was best at the cued location within an object, and discrimination performance decreased systematically as the within-object distance between the cue and the target was increased. The differences in sensitivity across the spatial extent of an object were quite large and highly robust. Discrimination accuracy at the cued location was nearly 20 percentage points higher than at far locations within the attended object. These results are consistent with studies of spatial attention showing that after a cue, attention is distributed in a graded manner, with highest sensitivity at the cue location and gradually decreasing sensitivity with increasing distance from the cue (Downing \& Pinker, 1985; Henderson, 1991; Henderson \& Macquistan, 1993; Mangun \& Hillyard, 1988).

Before discussing the within-object gradient finding further, it is important to discuss two alternative explanations for the basic gradient effect. First, it is possible that the gradient results reflected variability in the shift of attention to the cue. If attention was most often shifted to the cued region but occasionally shifted to the next closest region and rarely to more distant regions, then an apparent gradient could result from noisy attentional shifts. However, given that adjacent target locations were separated by $4.6^{\circ}$ in Experiments 1 and 2, attentional shifts to the cue would have to have been extraordinarily noisy indeed to produce the present pattern of results. In a similar study in which participants executed speeded saccades to the cued end of tube-like objects (Matsukura \& Hollingworth, unpublished data), eye movement landing position was highly precise, suggesting that the shift of covert attention before the saccade was generally accurate. In addition, a noisy-shift alternative has no means to account for the effect of same-different object in Experiment 1 and the modulation of the distance effect across a gap in Experiment 3. To generate these effects, a noisy shift hypothesis would need to further postulate that variability in the accuracy of the shift is influenced by object structure. Given strong converging evidence for attentional gradients in vision, the gradient account is certainly more parsimonious.

A second alternative account is that the basic gradient effect was generated by differences in the speed of shifting attention from the cued location to the target location on invalid trials, with shorter shifts completed more rapidly than longer shifts, generating facilitated target processing at invalid locations near the cue. However, the target was visible for only $70 \mathrm{~ms}$ before it was masked, making it unlikely that participants could have shifted attention to the target before it was masked under any conditions. In addition, the time required to shift attention has been found to be independent of the distance of the shift (Kwak, Dagenbach, \& Egeth, 1991; Murphy \& Eriksen, 1987; Sagi \& Julesz, 1985), making an attention shift account of the gradient effect unlikely.

In the present paradigm, the gradient of attention was limited to a relatively small region of the attended object and peaked at the cued location. It is interesting, then, to note that attention might not necessarily "fill" an object in the sense of generating increased perceptual sensitivity across the entire extent of the object. That is, there may be regions of an attended object that receive little or no facilitation relative to other objects or locations in the visual field. This may be particularly relevant for large objects (as used in the present experiments). If spatial attention is to be considered a limited pool of resources that can be distributed over the visual field, the size of the object could be an important factor in governing the extent to which attention "fills" an object and the density of the attentional distribution within the object. Consistent with this possibility, Davis et al. (2000) have observed a direct relationship between object size (i.e., area) and the efficiency of the perceptual processing of object features. In addition, attention may not have come to "fill" the object because participants were cued to a spatially localized object region. If the entire object had been cued, it is quite likely that we would have observed a more uniform distribution of attention across the object. Further inferences about the metric properties of the gradient of spatial attention over objects will require systematic manipulation of the many variables that could influence that distribution (e.g., object size, shape, the salience of object boundaries, part structure, cue type, task demands, and so on).

Our assessment of the gradient of attention in the present study probed the distribution of attention at one point in time (approximately $200 \mathrm{~ms}$ after the cue). This raises questions about the dynamics of attentional allocation and whether that allocation evolves systematically. Some evidence suggests that with extended viewing, attention might come to be peaked at the center of an object, even when the end of an object is cued. In a multiple-object tracking study, Alvarez and Scholl (2005) cued the ends of a subset of bars to be tracked. During tracking, participants reported the appearance of small dots on the bars. Detection accuracy was highest when the dot appeared in the center of an object, and not at the cued end, suggesting that the center had become preferentially selected for the purpose of tracking. In addition, eye movements to objects often land at the center of gravity of the object (e.g., Kowler \& Blaser, 1995; Melcher \& Kowler, 1999), which is consistent with the idea that covert attention (before the saccade) 
comes to be concentrated at the center of the object. However, these center-of-gravity effects have been tested under timing conditions much longer than those in the present experiments. Participants are typically instructed to take as long as they need to generate a saccade, and saccade latencies are often in the range of $1000 \mathrm{~ms}$; rapidly generated saccades show only minimal effects of object center of gravity (Matsukura \& Hollingworth, unpublished data). In the present experiments, attention certainly might have been distributed more uniformly through the object, and perhaps even concentrated in the center, if a longer delay between the cue and the target had been used. However, it is also the case that participants had strong incentive to keep attention concentrated at the cued location, so it is possible that participants were able to exert some degree of strategic control over how attention was distributed within the object. The question of whether the dynamic spreading of attention through an object is automatic or can be controlled will be a key topic for subsequent work.

\section{The Interaction Between Object Boundaries and the Gradient of Attention}

Several studies have illustrated the importance of object boundaries and local contours on attention to objects (Avrahami, 1999; Behrmann, Zemel, \& Mozer, 1998; Marino \& Scholl, 2005; Marrara \& Moore, 2003; Moore, Yantis, \& Vaughan, 1998; Vecera, Behrmann, \& Filapek, 2001; Watson \& Kramer, 1999). For example, within-object structure modulates the extent to which attention spreads to non-cued regions of an object (Matsukura \& Vecera, 2006; Vecera, Behrmann, \& Filapek, 2001; Watson \& Kramer, 1999), consistent with the general claim that object features interact with the distribution of attention. The present results extend this work by demonstrating that object structure interacts directly with the spatial gradient of attention. In Experiment 4, the gradient of attention across an object boundary was attenuated compared with the gradient of attention across the continuous extent of the object. The gradient was not eliminated across the boundary, however, suggesting that object boundaries may retard the spread of attention but do not necessarily eliminate it, consistent with recent evidence for attentional gradients in the immediate object surround (Kravitz \& Behrmann, 2008). ${ }^{6}$ This effect of object boundary provides direct support for the core hypothesis of the grouped-array account: higher-level object structure serves to constrain the spread of attention to a bounded, spatial array (Vecera \& Farah, 1994). Note that the object representations functional in guiding attention are likely to be generated after initial object segmentation and grouping. Not all physical contours are sufficient to limit the spread of attention, as attention can spread across the contour of an occluding object when the attended object is amodally completed behind it (Albrecht, List, \& Robertson, 2008; Moore, Yantis, \& Vaughan, 1998). In addition, the presence of a physical contour is not necessary to constrain the spread of attention, as a set of dots that imply a contour can generate object-based effects in the Egly et al. paradigm (Marrara \& Moore, 2003; see also Martinez et al., 2007). Thus, we believe that the representations serving to constrain attention are unlikely to be low-level image cues but rather higher-level, organized object representations. However, further work will be necessary to test this assumption within the context of the specific gradient and gap effects observed in the present study. By manipulating such factors as occlusion (Moore et al., 1998), it should be possible to determine whether constraints on the gradient of spatial attention are driven by higher-level representations of the objects in the display (e.g., post completion) or by the lower-level image features that are generated by the physical contours of the object.

At a broad level, effects of object structure on the spatial gradient of attention can be accommodated by computational models in which attention spreads across a spatially organized map (Mozer, 2002; Mozer \& Sitton, 1998). Such models instantiate a selective operation by limiting the number of attended locations that become active at any given time. Object perceptual structure provides bottom-up input to the attentional map, raising the activation of attended regions corresponding to visual objects, and input from object structure serves to constrain the spread of attention, limiting it to discrete object regions. In addition, as objects move, attention "sticks to" and "tracks" the objects, allowing these models to simulate dynamic object-centered effects, such as object-centered neglect in patients with parietal lobe damage (Mozer, 2002). Such models constitute a direct instantiation of the grouped-array hypothesis and provide a natural explanation of the gradient, object, and local discontinuity (gap) effects observed here.

Our account of Egly et al. (1994) effects in terms of a grouped array contrasts to some extent with the original proposal that such effects arise because attention must be switched between two discrete object representations on invalid trials (Egly et al.). One of the main contributions of the present study is the demonstration that two objects are not necessary to generate object-based effects. In Experiments 3 and 4, an effect of object structure was observed on perceptual sensitivity despite the fact that there was only one object in the display. This result is not explained naturally by the need to switch attention between two objects, as there was only one object present. It is, however, quite naturally explained by the grouped array approach, as object boundaries are proposed to lie at the heart of the effect. Further, the Egly et al. attention shift account is limited somewhat by the fact their original onset detection task did not logically require a shift of attention on invalid trials. Their targets were simple luminance transients. Luminance transients can be detected without focal attention, and thus participants could have detected and responded to the target on invalid trials without ever shifting attention to the target location, especially as they did not need to localize the target to respond. Of course, we cannot infer that participants do not, in practice, shift attention before the response in onset detection versions of the Egly et al. task, just that it is not required by the task. In the grouped array account of the original Egly et al. effect, the spatial distribution of attention within and across objects (i.e., graded and concentrated within the attended object) at the time of target onset modulates the efficiency of perceptual processing of the onset signal itself, generating differences in RT as a function of distance and same/different object. This account does not require shifts of attention at all.

\footnotetext{
${ }^{6}$ An alternative is that attention begins to spread immediately after the cue in a manner that is not constrained by object structure, and that object-structure subsequently modulates the distribution of attention, resulting in some lingering facilitation at locations not contained within the object. We thank an anonymous reviewer for suggesting this possibility.
} 
The effect of a gap in a single object in Experiment 3 was numerically no smaller than the effect in the standard, two-object condition of that experiment. This equivalence might be taken to suggest that the entire object effect in the Egly et al. (1994) paradigm can be attributed to a grouped array mechanism and that switching attention between objects plays little or no functional role. In addition, the gradient effects in the onset detection version of our paradigm suggest that a grouped array account can generalize to the type of simple detection task used originally by Egly et al. However, we think it is unlikely that grouped-array processes are the only means by which object-based effects can be generated in this type of paradigm. The basic method here was designed to eliminate the utility of attention shifts, with the very brief presentation of the target $(70 \mathrm{~ms})$ providing little opportunity to shift attention to an invalid target before the onset of the mask. It is quite likely that paradigms which require shifts of attention to invalid locations can generate an object-based effect above and beyond that attributable to grouped-array processes. This is particularly applicable to paradigms that present multiple targets within the two objects and require shifts of attention via serial, conjunction search through the objects (Hecht \& Vecera, 2007; Moore et al., 1998; Shomstein \& Yantis, 2004).

\section{Object Selection and Perceptual Sensitivity}

The present paradigm was designed to assess differences in perceptual sensitivity as a function of object and of distance from the cue. A pattern discrimination task was used instead of onset detection so as to minimize effects of response bias, which can make onset detection results difficult to interpret with regard to perceptual enhancement (Luck \& Vecera, 2002). The target was presented very briefly and masked, to eliminate the utility of attention shifts and thus provide a direct measure of perceptual efficiency at the time that the target appeared. There was only one target, which eliminated the need to search through the display, and there was only one masked location, which eliminated any uncertainty in target location and thus eliminated any potential effect of cuing on the reduction of decision uncertainty (Palmer, 1994; Shiu \& Pashler, 1994). In sum, the paradigm included all of the central features of spatial attention paradigms that have found direct evidence of cuing effects on perceptual sensitivity (e.g., Henderson, 1996; Pestilli \& Carrasco, 2005).

Although the original conceptualizations of the grouped array theory did not discuss perceptual enhancement directly, the effects of object on perceptual sensitivity are consistent with the grouped array assumption that midlevel, preattentive perceptual grouping processes influence the distribution of spatial attention in a bottom-up manner. In addition, recent reviews (Mozer \& Vecera, 2005a) and computational models (Mozer, 2002; Vecera \& O'Reilly, 1998) of the grouped array process support perceptual enhancement as the consequence of selection. For example, the Vecera and O'Reilly model of figure-ground assignment implements the effects of salient perceptual groups (figures) as influencing the efficiency of perceptual processing within that group. In addition, Mozer's (2002) model of object-centered neglect also assumes that attended objects are perceptually enhanced.

\section{Conclusion}

The present study establishes a theoretical and empirical bridge between research on object and spatial attention. The results ground and expand the grouped-array hypothesis, which holds that object selection at a perceptual level is accomplished through the influence of object image structure on the distribution of spatial attention. In the present experiments, we observed a robust spatial gradient of attention distributed through an attended object, and we observed that the modulation of this gradient by object structure can generate object-based effects commonly observed in the literature. Thus, attention to locations and attention to objects need not be dichotomous; object representations and spatial attention interact dynamically, and object-based effects can be generated by an inherently spatial mechanism.

\section{References}

Albrecht, A. R., List, A., \& Robertson, L. C. (2008). Attentional selection and the representation of holes and objects. Journal of Vision, 8, 1-10.

Alvarez, G. A., \& Scholl, B. J. (2005). How does attention select and track spatially extended objects? New effects of attentional concentration and amplification. Journal of Experimental Psychology: General, 134, 461476.

Atchley, P., \& Kramer, A. F. (2001). Object and space-based attentional selection in three-dimensional space. Visual Cognition, 8, 1-32.

Avrahami, N. (1999). Objects of attention, objects of perception. Perception \& Psychophysics, 61, 1604-1612.

Awh, E., Dhaliwal, H., Christensen, S., \& Matsukura, M. (2001). Evidence for two components of object-based selection. Psychological Science, 12, 329-334.

Behrmann, M., Zemel, R. S., \& Mozer, M. C. (1998). Object-based attention and occlusion: Evidence from normal participants and a computational model. Journal of Experimental Psychology: Human Perception and Performance, 24, 1011-1036.

Carrasco, M., Penpeci-Talgar, C., \& Eckstein, M. (2000). Spatial covert attention increases contrast sensitivity across the CSF: Support for signal enhancement. Vision Research, 40, 1203-1215.

Cheal, M. L., \& Lyon, D. R. (1991). Central and peripheral precuing of forced-choice discrimination. Quarterly Journal of Experimental Psychology, 43A, 859-880.

Chen, Z., \& Cave, K. R. (2006). Reinstating object-based attention under positional certainty: The importance of subjective parsing. Perception \& Psychophysics, 68, 992-1003.

Cole, G., Gellatly, A., \& Blurton, A. (2001). Effect of object onset on the distribution of visual attention. Journal of Experimental Psychology: Human Perception and Performance, 27, 1356-1368.

Davis, G., Driver, J., Pavani, F., \& Shepherd, A. (2000). Reappraising the apparent costs of attending to two separate visual objects. Vision Research, 40, 1323-1332.

Downing, C. J., \& Pinker, S. (1985). The spatial structure of visual attention. In M. I. Posner \& O. S. Marin (Ed.), Mechanisms of attention: Attention and performance XI (pp. 171-187). Hillsdale, NJ: Erlbaum.

Drummond, L., \& Shomstein, S. (2010). Object-based attention: Shifting or uncertainty? Attention, Perception, \& Psychophysics, 72, 1743-1755.

Duncan, J. (1984). Selective attention and the organization of visual information. Journal of Experimental Psychology: General, 113, 501517.

Egly, R., Driver, J., \& Rafal, R. D. (1994). Shifting visual attention between objects and locations: Evidence from normal and parietal lesion subjects. Journal of Experimental Psychology: General, 123, 161-177.

He, X., Fan, S. L., Zhou, K., \& Chen, L. (2004). Cue validity and object-based attention. Journal of Cognitive Neuroscience, 16, 10851097. 
Hecht, L. N., \& Vecera, S. P. (2007). Attentional selection of complex objects: Joint effects of surface uniformity and part structure. Psychonomic Bulletin \& Review, 14, 1205-1211.

Henderson, J. M. (1991). Stimulus discrimination following covert attentional orienting to an exogenous cue. Journal of Experimental Psychology: Human Perception and Performance, 17, 91-106.

Henderson, J. M. (1996). Spatial precues affect target discrimination in the absence of visual noise. Journal of Experimental Psychology: Human Perception and Performance, 22, 780-787.

Henderson, J. M., \& Hollingworth, A. (1999). High-level scene perception. Annual Review of Psychology, 50, 243-271.

Henderson, J. M., \& Macquistan, A. D. (1993). The spatial distribution of attention following an exogenous cue. Perception \& Psychophysics, 53, 221-230.

Hillyard, S. A., Vogel, E. K., \& Luck, S. J. (1998). Sensory gain control (amplification) as a mechanism of selective attention: Electrophysiological and neuroimaging evidence. Philosophical Transactions of the Royal Society of London Series B-Biological Sciences, 353, 1257-1270.

Kowler, E., \& Blaser, E. (1995). The accuracy and precision of saccades to small and large targets. Vision Research, 35, 1741-1754.

Kramer, A. F., \& Jacobson, A. (1991). Perceptual organization and focused attention: The role of objects and proximity in visual processing. Perception \& Psychophysics, 50, 267-284.

Kramer, A. F., Weber, T. A., \& Watson, S. E. (1997). Object-based attentional selection-Grouped-arrays or spatially invariant representations? Comment on Vecera and Farah (1994). Journal of Experimental Psychology: General, 126, 3-13.

Kravitz, D. J., \& Behrmann, M. (2008). The space of an object: Object attention alters the spatial gradient in the surround. Journal of Experimental Psychology: Human Perception and Performance, 34, 298-309.

Kwak, H. W., Dagenbach, D., \& Egeth, H. (1991). Further evidence for a time-independent shift of the focus of attention. Perception \& Psychophysics, 49, 473-480.

LaBerge, D., \& Brown, V. (1989). Theory of attentional operations in shape identification. Psychological Review, 96, 101-124.

Lamy, D., \& Egeth, H. (2002). Object-based selection: The role of attentional shifts. Perception \& Psychophysics, 64, 52-66.

Land, M. F., \& Hayhoe, M. (2001). In what ways do eye movements contribute to everyday activities? Vision Research, 41, 3559-3565.

Luck, S. J., Hillyard, S. A., Mouloua, M., \& Hawkins, H. L. (1996). Mechanisms of visual-spatial attention: Resource allocation or uncertainty reduction? Journal of Experimental Psychology: Human Perception and Performance, 22, 725-737.

Luck, S. J., \& Vecera, S. P. (2002). Attention: From tasks to mechanisms. In S. Yantis (Ed.), Stevens' handbook of experimental psychology: Vol. 1. Sensation and perception (pp. 235-286). New York: Wiley.

Mangun, G. R., \& Hillyard, S. A. (1988). Spatial gradients of visualattention: Behavioral and electrophysiological evidence. Electroencephalography and Clinical Neurophysiology, 70, 417-428.

Marino, A. C., \& Scholl, B. J. (2005). The role of closure in defining the "objects" of object-based attention. Perception \& Psychophysics, 67, $1140-1149$

Marrara, M. T., \& Moore, C. M. (2003). Object-based selection in the two-rectangles method is not an artifact of the three-sided directional cue. Perception \& Psychophysics, 65, 1103-1109.

Martinez, A., Ramanathan, D. S., Foxe, J. J., Javitt, D. C., \& Hillyard, S. A. (2007). The role of spatial attention in the selection of real and illusory objects. Journal of Neuroscience, 27, 7963-7973.

Martinez, A., Teder-Salejarvi, W., \& Hillyard, S. A. (2007). Spatial attention facilitates selection of illusory objects: Evidence from event-related brain potentials. Brain Research, 1139, 143-152.

Martinez, A., Teder-Salejarvi, W., Vazquez, M., Molholm, S., Foxe, J. J., Javitt, D. C., ... Hillyard, S. A. (2006). Objects are highlighted by spatial attention. Journal of Cognitive Neuroscience, 18, 298-310.
Matsukura, M., \& Vecera, S. P. (2006). The return of object-based attention: Selection of multiple-region objects. Perception \& Psychophysics, 68, 1163-1175.

Matsukura, M., \& Vecera, S. P. (2009). Interference between object-based attention and object-based memory. Psychonomic Bulletin \& Review, 16, $529-536$.

Melcher, D., \& Kowler, E. (1999). Shapes, surfaces and saccades. Vision Research, 39, 2929-2946.

Moore, C. M., Yantis, S., \& Vaughan, B. (1998). Object-based visual selection: Evidence from perceptual completion. Psychological Science, 9. $104-110$

Mozer, M. C. (2002). Frames of reference in unilateral neglect and visual perception: A computational perspective. Psychological Review, 109, $156-185$.

Mozer, M. C., \& Sitton, M. (1998). Computational modeling of spatial attention. In H. Pashler (Ed.), Attention (pp. 341-393). London: Psychology Press.

Mozer, M. C., \& Vecera, S. P. (2005a). Object- and space-based attention. In L. Itti, G. Rees \& J. K. Tsotsos (Eds.), Neurobiology of attention (pp. 130-134). New York: Elsevier.

Mozer, M. C., \& Vecera, S. P. (2005b). Object-based and space-based attention. In L. Itti, G. Rees \& J. K. Tsotsos (Eds.), Neurobiology of Attention (pp. 130-134). New York: Elsevier.

Müller, H. J., \& Rabbit, P. M. (1989). Reflexive and voluntary orienting of visual attention: Time course of activation and resistance to interruption. Journal of Experimental Psychology: Human Perception and Performance, $15,315-330$.

Müller, N. G., \& Kleinschmidt, A. (2003). Dynamic interaction of objectand space-based attention in retinotopic visual areas. Journal of Neuroscience, 23, 9812-9816.

Murphy, T. D., \& Eriksen, C. W. (1987). Temporal changes in the distribution of attention in the visual field in response to precues. Perception \& Psychophysics, 42, 576-586.

Palmer, J. (1994). Set-size effects in visual search: The effect of attention is independent of the stimulus for simple tasks. Vision Research, 34, 1703-1721.

Pestilli, F., \& Carrasco, M. (2005). Attention enhances contrast sensitivity at cued and impairs it at uncued locations. Vision Research, 45, 18671875.

Posner, M. I. (1980). Orienting of attention. Quarterly Journal of Experimental Psychology, 32, 3-25.

Posner, M. I., Snyder, C. R. R., \& Davidson, B. J. (1980). Attention and the detection of signals. Journal of Experimental Psychology: General, 109, $160-174$.

Richard, A. M., Lee, H., \& Vecera, S. P. (2008). Attentional spreading in object-based attention. Journal of Experimental Psychology: Human Perception and Performance, 34, 842-853.

Sagi, D., \& Julesz, B. (1985). Fast noninertial shifts of attention. Spatial Vision, 1, 141-149.

Schneider, W., Eschmann, A., \& Zuccolotto, A. (2002). E-Prime user's guide. Pittsburgh, PA: Psychology Software Tools, Inc.

Scholl, B. J. (2001). Objects and attention: The state of the art. Cognition, $80,1-46$.

Shiu, L.-P., \& Pashler, H. (1994). Negligible effect of spatial precuing on identification of single digits. Journal of Experimental Psychology: Human Perception and Performance, 20, 1037-1054.

Shomstein, S., \& Yantis, S. (2002). Object-based attention: Sensory modulation or priority setting? Perception \& Psychophysics, 64, 41-51.

Shomstein, S., \& Yantis, S. (2004). Configural and contextual prioritization in object-based attention. Psychonomic Bulletin \& Review, 11, 247-253.

Valdes-Sosa, M., Bobes, M. A., Rodriguez, V., \& Pinilla, T. (1998). Switching attention without shifting the spotlight object-based atten- 
tional modulation of brain potentials. Journal of Cognitive Neuroscience, 10, 137-151.

Vecera, S. P. (1994). Grouped locations and object-based attention: Comment on Egly, Driver, and Rafal (1994). Journal of Experimental Psychology: General, 123, 316-320.

Vecera, S. P., Behrmann, M., \& Filapek, J. C. (2001). Attending to the parts of a single object: Part-based selection limitations. Perception \& Psychophysics, 63, 308-321.
Vecera, S. P., \& Farah, M. J. (1994). Does visual attention select objects or locations? Journal of Experimental Psychology: General, 123, 146-160.

Vecera, S. P., \& O'Reilly, R. C. (1998). Figure-ground organization and object recognition processes: An interactive account. Journal of Experimental Psychology: Human Perception and Performance, 24, 441-462.

Watson, S. E., \& Kramer, A. F. (1999). Object-based visual selective attention and perceptual organization. Perception \& Psychophysics, 61, $31-49$.

\section{Appendix}

\section{Mean Reaction Time Data (ms) for Each of the Masked Discrimination Experiments}

Experiment 1

\begin{tabular}{cccc}
\hline \multicolumn{3}{c}{ Cue-target distance/object } \\
\hline 0 (Valid) & 1 (Near) & 2 (Far, same object) & 2 (Far, different object) \\
\hline 669 & 795 & 793 & 788 \\
\hline
\end{tabular}

Experiment 2

\begin{tabular}{ccccc}
\hline \multicolumn{5}{c}{ Cue-target distance } \\
\hline 0 (Valid) & 1 & 2 & 3 & 4 \\
\hline 669 & 735 & 751 & 771 & 760 \\
\hline
\end{tabular}

Experiment 3

\begin{tabular}{ccccccc}
\hline \multicolumn{3}{c}{ One object } & & \multicolumn{3}{c}{ Two objects } \\
\cline { 1 - 1 } Valid & Invalid (along object) & Invalid (across gap) & & Valid & Invalid (along object) & Invalid (across gap) \\
\hline 603 & 682 & 691 & & 591 & 663 & 678 \\
\hline
\end{tabular}

Experiment 4

\begin{tabular}{|c|c|c|c|c|c|c|c|}
\hline \multicolumn{8}{|c|}{ Cue-target distance } \\
\hline \multicolumn{4}{|c|}{ Along object } & \multicolumn{4}{|c|}{ Across gap } \\
\hline 0 (Valid) & 1 & 2 & 3 & 4 & 1 & 2 & 3 \\
\hline 646 & 672 & 691 & 681 & 664 & 726 & 670 & 716 \\
\hline
\end{tabular}

Received January 1, 2011

Revision received April 26, 2011

Accepted April 26, 2011 